\title{
Coupled tomography and distinct-element-method approach to exploring the granular media microstructure in a jamming hourglass
}

\author{
M. Tsukahara, ${ }^{1}$ S. Mitrovic, ${ }^{2}$ V. Gajdosik, ${ }^{2}$ G. Margaritondo, ${ }^{2}$ L. Pournin, ${ }^{1}$ M. Ramaioli, ${ }^{1}$ D. Sage, ${ }^{3}$ Y. Hwu, ${ }^{4}$ M. Unser, ${ }^{3}$ \\ and Th. M. Liebling ${ }^{1}$ \\ ${ }^{1}$ Mathematics Institute, École Polytechnique Fédérale de Lausanne, CH-1015 Lausanne, Switzerland \\ ${ }^{2}$ Institut de Physique de la Matiere Complexe, École Polytechnique Fédérale de Lausanne, CH-1015 Lausanne, Switzerland \\ ${ }^{3}$ Biomedical Imaging Group, École Polytechnique Fédérale de Lausanne, CH-1015 Lausanne, Switzerland \\ ${ }^{4}$ Institute of Physics, Academia Sinica, Nankang, Taipei 115. Taiwan
}

(Received 5 September 2007; revised manuscript received 18 March 2008; published 25 June 2008)

\begin{abstract}
We describe an approach for exploring microscopic properties of granular media that couples X-ray microtomography and distinct-element-method (DEM) simulations through image analysis. We illustrate it via the study of the intriguing phenomenon of instant arching in an hourglass (in our case a cylinder filled with a polydisperse mixture of glass beads that has a small circular shutter in the bottom). X-ray tomography provides three-dimensional snapshots of the microscopic conditions of the system both prior to opening the shutter, and thereafter, once jamming is completed. The process time in between is bridged using DEM simulation, which settles to positions in remarkably good agreement with the x-ray images. Specifically designed image analysis procedures accurately extract the geometrical information, i.e., the positions and sizes of the beads, from the raw x-ray tomographs, and compress the data representation from initially 5 gigabytes to a few tens of kilobytes per tomograph. The scope of the approach is explored through a sensitivity analysis to input data perturbations in both bead sizes and positions. We establish that accuracy of size-much more than positionestimates is critical, thus explaining the difficulty in considering a mixture of beads of different sizes. We further point to limits in the replication ability of granular flows away from equilibrium; i.e., the difficulty of numerically reproducing chaotic motion.
\end{abstract}

DOI: 10.1103/PhysRevE.77.061306

PACS number(s): 45.70.Cc, 42.30.Wb

\section{INTRODUCTION}

Due to their pervasiveness in nature, technology, and everyday life, granular media have been the object of extensive research using both experiments and numerical models. Several studies compare the adequacy of these two approaches for characterizing bulk or macroscopic properties of granular media. This paper presents an approach to studying microscopic properties of granular media that consists in coupling $\mathrm{x}$-ray tomography and distinct-element-method (DEM) simulations (tomo-DEM) through image analysis.

We introduce the procedure, technical details, and limitations of this coupled tomo-DEM technique through an application to the study of the intriguing phenomenon of arching that instantly jams the flow of-in our case polydispersegrains in a cylinder upon the opening of a small bottom circular shutter. This phenomenon is a benchmark for the study of near-equilibrium granular dynamics, and has a broad impact on a variety of practical issues such as silo blocking [1]. In the present study, x-ray tomography provided three-dimensional (3D) snapshots of the initial microscopic conditions of the system both prior to opening the shutter, and upon reaching the end of the jamming process once the shutter was opened. The process time in between was bridged using DEM simulation, which settled to positions in remarkably good agreement with the $\mathrm{x}$-ray images. An overview of the methodology is given in Fig. 1 .

The results also underscore the power of synchrotron $\mathrm{x}$-ray tomography in analyzing granular systems on a microscopic scale. This is a significant step forward: threedimensional nonintrusive viewing of the inside of porous or granular media is indeed very valuable. Recent progress was made possible by magnetic resonance imaging and $\mathrm{x}$-ray computed tomography and microtomography, whereas researchers were previously forced to mechanically disassemble the media [2-5]. In recent years, 3D imaging was used to investigate the geometrical properties of void space for a better insight into flow properties [6-10], granular media packing [11-15], or foam structure [16]. Seidler et al. [11] identified the central positions and local connectivity of 2000 monodisperse glass spheres from x-ray microtomography data using edge location and the Hough transform. Richard et al. [12] found the size and location of 15000 glass beads by $\mathrm{x}$-ray microtomography while analyzing granular systems undergoing compaction. Aste et al. [13] analyzed two 150 000-bead samples and four 35 000-bead samples by $\mathrm{x}$-ray computed tomography and a convolution method. Fu et al. [15] investigated 2000 particles using x-ray microtomography and a watershed-based segmentation algorithm and showed that packing systems generated by the DEM are consistent with the structural measurements made by $\mathrm{x}$-ray microtomography. Despite these significant advances, efficient extraction from tomographic images of the microscopic geometric information such as the sizes of voids or the grain radii and positions still constitutes a challenge. Our study proposes an effective computerized strategy.

Various types of distinct-element granular media simulation models have been proposed in the literature, like, for example, molecular dynamics models $[17,18]$, contact dynamics models [19], event-driven models [20,21], or Monte Carlo simulations [22]. Pournin et al. [23] and Ramaioli et al. [24] demonstrate the use of the DEM for reproducing 


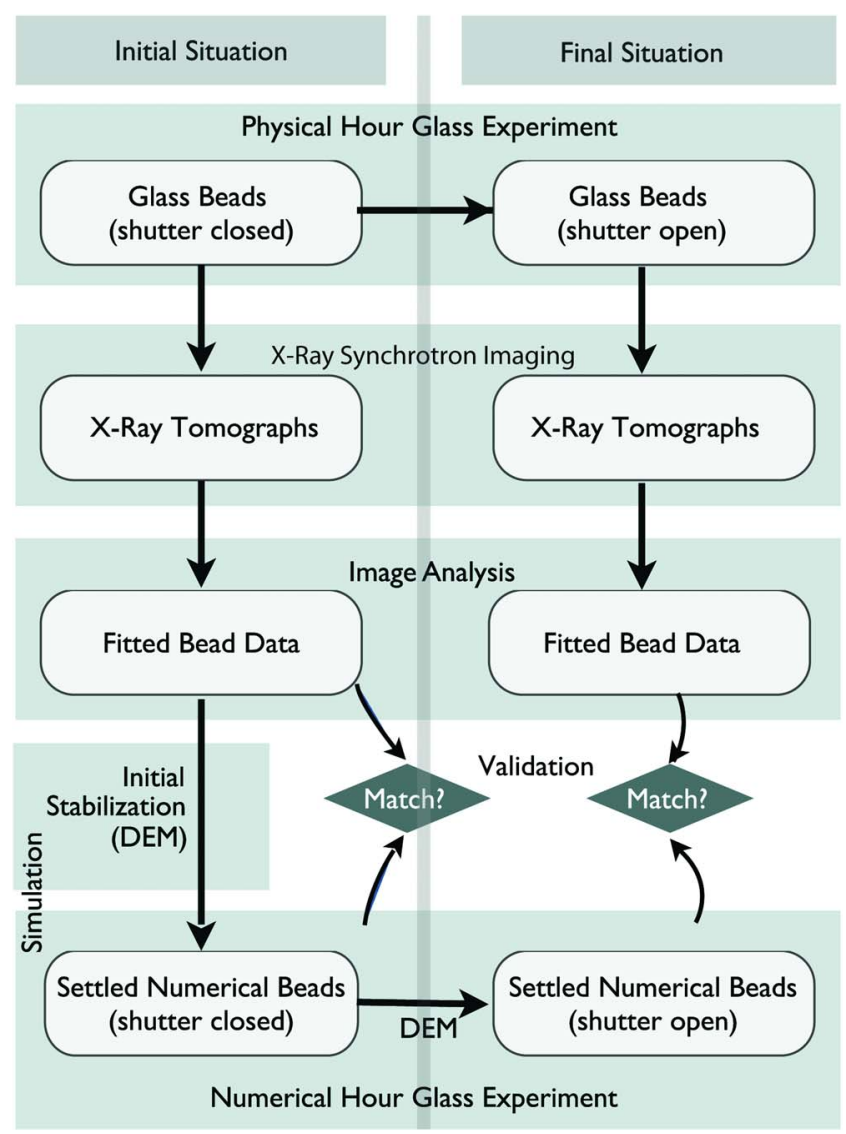

FIG. 1. (Color online) Combining Synchrotron x-ray tomography and DEM: an overview.

granular phenomena such as crystallization and segregation. In [1], Pournin et al. also study jamming in an hourglass both experimentally and using DEM simulations. They show that for given system parameters (container geometry and bead granulometry) DEM enables an accurate estimate of the jamming probability.

Here we go a large step further: not only do we predict jamming for particular real initial configurations, but we also accurately reproduce the actual jammed configurations. Our technique thus couples soft-sphere DEM simulation with $\mathrm{X}$-ray tomography to gain better insight into the microstructure of granular media and near-equilibrium granular dynamics.

The remainder of this paper is organized as follows: First we introduce the x-ray synchrotron hourglass imaging experiment. Next we describe and discuss the ensuing elaborate image analysis procedures implemented to extract accurate positions and sizes of the beads from the raw images, suitable to be used in numerical simulations, thereby compressing the data representation from initially some 5 gigabytes to just a few tens of kilobytes per tomograph. Then we present numerical hourglass experiments using DEM simulation (in two phases, the first needed to stabilize the numerical beads' initial positions with the shutter closed, the second to replicate the flow in the hourglass upon opening the shutter) and discuss the results. Finally, in a sensitivity analysis to data perturbations of size and position of the numerical beads, we show that accurate bead size estimates are crucial for suc-

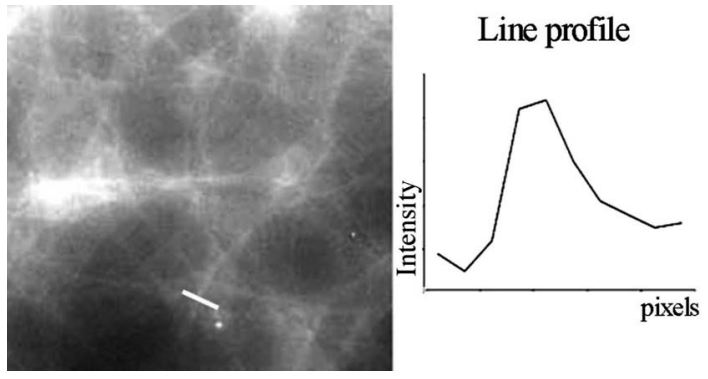

FIG. 2. Tuning the sample-detector distance for optimal contrast enhancement in 2D projection: the line intensity profile (white mark) through the glass-air interface shows a peak of higher intensity corresponding to the refraction-based effects.

cess. Interestingly, position perturbation energy is equilibrium restoring while size perturbation energy irreversibly destroys the equilibrium, even when average bead volume is kept constant. The analysis also points to the limits of the tomo-DEM approach concerning the replication of granular flows far from equilibrium, thus exhibiting the difficulty of numerically reproducing chaotic motion.

\section{THE EXPERIMENT}

Edge-enhanced x-ray microtomography using broadband synchrotron radiation is a powerful nondestructive tool for research on packing and jamming in granular systems. Standard absorption tomography is based on the 3D determination of the linear attenuation coefficient and is related only to the imaginary part of the complex refraction index. Thanks to the high spatial coherence of the synchrotron $x$ rays we can use effects based on the real part of refraction index to enhance the edge visibility [25]. In an x-ray beam glass beads act as weak divergent lenses and phase effects are visible on the glass/air interface of each sphere as concentric white bands (Fig. 2). Even with polychromatic X-ray radiation, this effect contributes remarkably to absorption contrast and could be optimized by simply changing in the propagation (sample to detector) distance. In contrast, the air defects inside the glass are enhanced by focusing_acting as weak convergent lenses.

All tomography experiments were performed on the white-light imaging beamline BLO1A at the National Synchrotron Research Center, Hsinchu, Taiwan as described in [26]. Bending-magnet synchrotron radiation with a continuous spectrum (photon energy 5-20 keV) was used in a lensless configuration (Fig. 3).

After passing through the sample, the $\mathrm{x}$ rays were converted to visible light by a $\mathrm{CdWO}_{4}$ crystal scintillator. The image was then magnified with an optical microscope and captured by a charge-coupled device camera. This approach gets the advantage of the entire beam size and makes possible imaging millimeter-size specimens with micrometer lateral resolution. By adjusting the sample-detector distance for a limited edge-enhancement 3D volume, tomographic reconstruction based on a sequence of projection images taken at different angles is feasible without introducing serious artifacts [27]. A common filtered back projection implemented 


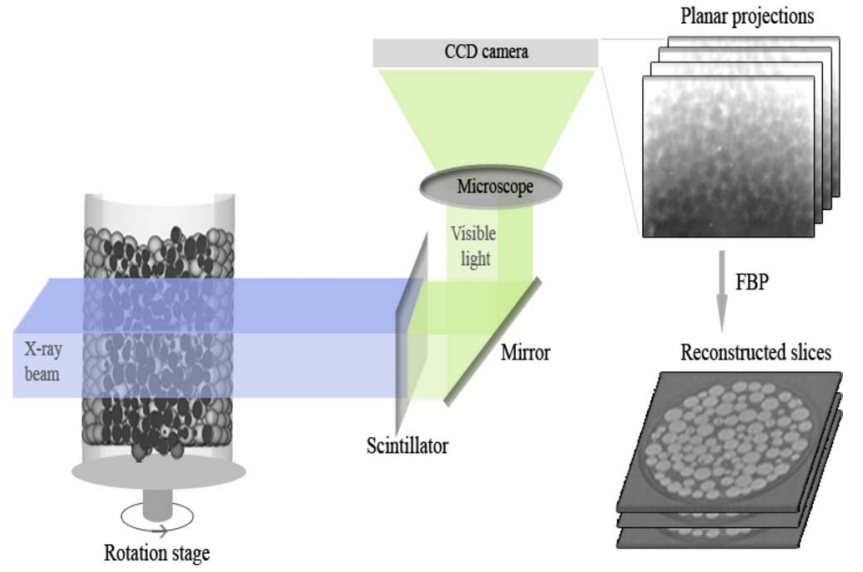

FIG. 3. (Color online) Scheme of the synchrotron microtomography imaging setup.

in interactive data language was used for this reconstruction. Nine different experiments (Table I) were conducted on two sets of spherical glass beads (Assistent-Precision, Germany). Set 1 contained mixed-size individually selected beads avoiding aspherical shapes and other fabrication artifacts. Set 2 underwent the same procedure and was sieved, keeping only particles with radii within the range $0.2-0.25 \mathrm{~mm}$.

A boron nitride cylindrical container was selected because of its resistance to radiation damage (Fig. 4). The container had two interchangeable circular openings with diameters $B=1.5$ and $2.0 \mathrm{~mm}$. The bottom of the opening was equipped with a paper sheet shutter which was positioned under an interchangeable bottom piece at offset $C=1.0 \mathrm{~mm}$ to the receptacle bottom.

Projection images were first taken with the bottom aperture closed to obtain the initial conditions for DEM simulations. Then the shutter was opened, letting the beads flow. A second projection image set was acquired after the bead flow jammed and an arch was formed. All tomography sets were obtained for three adjacent vertical positions and merged to cover a volume that contained around 1000 particles for set 1 and 2000 particles for set 2 .

TABLE I. Description of the experimental setup. Expt.: Experiment label (first number indicates if hole radius is $\sim$ three or four times the average bead radius, letter $b$ indicates sieved bead experiments, second number indicates experiment number); BS: bead sizes (1 mixed; 2 sieved); $\langle r\rangle$ : average radius; SD: standard deviation; HR: hole radius.

\begin{tabular}{ccccc}
\hline \hline Expt. & BS & $\langle r\rangle(\mu \mathrm{m})$ & $\mathrm{SD}(\mu \mathrm{m})$ & $\mathrm{HR}(\mathrm{mm})$ \\
\hline $3 \_1$ & 1 & 275 & 27 & 0.75 \\
$3 \_2$ & 1 & 275 & 27 & 0.75 \\
$3 \_3$ & 1 & 275 & 27 & 0.75 \\
$4 \_5$ & 1 & 275 & 27 & 1.0 \\
$3 b \_1$ & 2 & 230 & 16 & 0.75 \\
$36 \_3$ & 2 & 230 & 16 & 0.75 \\
$4 b \_1$ & 2 & 230 & 16 & 1.0 \\
$4 b \_2$ & 2 & 230 & 16 & 1.0 \\
$4 b \_3$ & 2 & 230 & 16 & 1.0 \\
\hline \hline
\end{tabular}

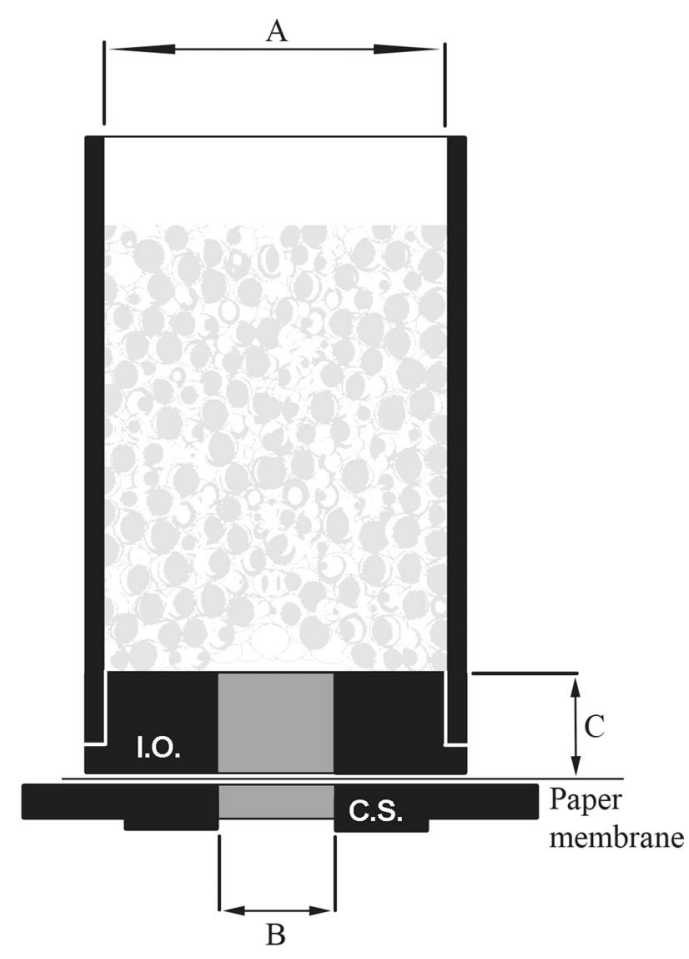

FIG. 4. The container (diameter $A=6.0 \mathrm{~mm}$ ) was drilled from one block of boron nitride and has interchangeable openings (IO) (diameter $B=1.5 \mathrm{~mm}$ and $2.0 \mathrm{~mm}$ ). A paper shutter was positioned under the container at offset $C=1.0 \mathrm{~mm}$ from its bottom. The container was mounted on the top of a metal sample holder CS.

\section{IMAGE ANALYSIS}

\section{A. The processing procedure}

We now describe our procedure to obtain the positions and radii of the various observed particles from the raw tomography images. The raw tomograph [Fig. 5(a)] had 1599 pixels in the $x$ and $y$ horizontal directions and approximately 1985 pixels in the $z$ direction, occupying nearly 5 gigabytes of computer memory. Each pixel corresponds to a cube size of $4 \times 4 \times 4 \mu \mathrm{m}^{3}$. Hence, the field of view is of size 6.396 $\times 6.396 \times 7.94 \mathrm{~mm}^{3}$. The large amount of data is one of the complicating factors of the image analysis along with other problems such as (1) low contrast: the void (air) and the glass beads have similar x-ray absorption factors (on a scale of 256 gray tones, the average void gray is 145 while that of the beads is 160); (2) noise: the contrast-to-noise ratio (CNR) is typically $0.56 \mathrm{~dB}$, and thus very poor; (3) Newton rings [Fig. 5(b)]; (4) splashes [Fig. 5(c)]; (5) air bubbles in the beads [Fig. 5(e)]; (6) nonuniformity of the intensity along the $z$ axis.

Furthermore, in spite of our efforts to select spherical beads, some nonspherical particles were still present [Fig. $5(d)$. Our objective here was to obtain accurate estimates of the positions and radii of all observed beads. Since standard image-analysis software cannot treat beads in the above problematic conditions, we had to develop our own procedure with algorithms and the corresponding software, as described below.

The main processing steps are preprocessing of the raw images, preliminary detection of spheres that fit the beads 

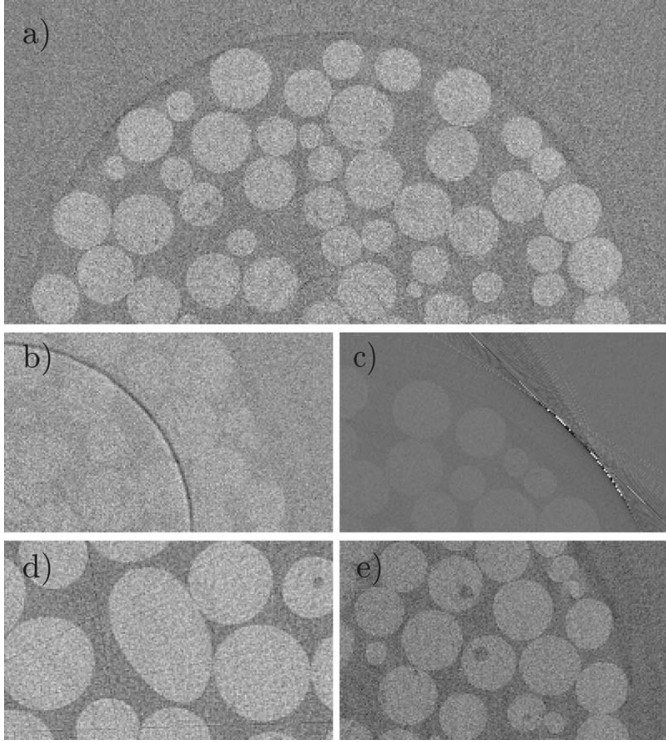

FIG. 5. Raw tomograph (and artifacts) used as input for image analysis: (a) Half of a horizontal cut ( $z$ layer); (b) Newton ring; (c) splashes; (d) asphericity; (e) air bubbles.

(sphere fittings) using a 3D watershed, and, third, optimization of the preliminary sphere fittings. A multiresolution approach was chosen, i.e., the size of the images was varied so that the computer could handle the memory requirements of the different processes.

The preprocessing consisted in smoothing the images to reduce the noise and in applying an adaptative thresholding procedure along the $z$ layers. The threshold value was independently computed for every layer to compensate the nonuniformities along the $z$ axis. However, this treatment was not sufficient to free the images of the traces of Newton rings and air bubbles, and manual corrections as well as imageprocessing morphological operations (dilation, erosion) were applied to further clean the images of artifacts [Fig. 6(a)]. This preprocessing was applied to the original images reduced by $50 \%$ in $x, y$, and $z$ directions.
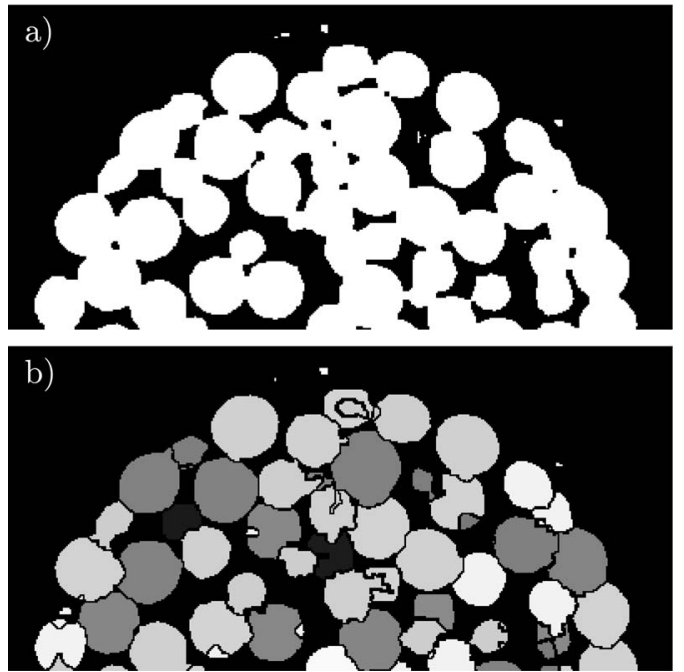

FIG. 6. Half of (a) a preprocessed $z$ layer and (b) a watershed $z$ layer.
The detection step consisted in segmenting the binary volume data set into regions and then fitting spheres to the found regions. Watershed by immersion [28] was used for this segmentation. This algorithm introduced for 2D images by Vincent and Soille [29] has been employed in various domains including powder and granular matter $[15,30]$. Here, we have developed and implemented its extension to the 3D case. Specifically, we first created a distance map transform of the binary volume by applying successive erosions. The result of the distance map transform is an image where the gray level is the distance from the closest boundary pixel. We then applied the immersion algorithm to obtain regions as shown in Fig. 6(b).

The result of the watershed segmentation was good for a large portion of the spheres. However, segmentation by the watershed algorithm is affected by errors of three types: (1) two or more regions can be found for only one bead (oversegmentation); (2) one region can be found for two or more beads (undersegmentation); (3) the boundary between two beads can be badly estimated (bad cut). The first error can be corrected algorithmically since the regions of an oversegmentation interpenetrate each other so that the corresponding sphere fittings have large overlaps. It is thus possible to merge the sphere fittings so that the final sphere is a correct estimation of the bead.

Correcting for undersegmentation and bad-cut errors is much trickier and we therefore resorted to manually editing these. For the computer processing of the other beads, the sphere fitting radius was defined as the radius of a sphere with the same volume as the watershed region of the bead. The sphere fitting position was defined as the average location of pixels in that region.

The watershed algorithm being demanding as far as computer memory is concerned, the detection stage was applied to images reduced in size by $75 \%$ with respect to the original images in the $x, y$, and $z$ directions. This causes a certain loss of information: it was indeed necessary to return to $50 \%$ reduced images for more precise sphere fitting. To achieve this, starting from the sphere fitting found in the detection stage and the $50 \%$ reduced binary images obtained after preprocessing, an optimization program was implemented. We looked for the largest sphere contained in the bead. Since the beads are not far from being spherical this is reasonable. The optimum sphere was found by an ad hoc gradient method. Figure 7 shows a comparison of sphere fittings before and after the optimization.

\section{B. Results and validation of the image-processing procedure}

We implemented the whole process as a plugin series for IMAGEJ [31], a general purpose image-processing software (public domain license) and ran the software on a Mac computer. 3D tomographic images corresponding to initial and final configurations were processed for seven of the nine experiments detailed in Table I. For experiments $4 b_{-} 1$ and $4 b \_2$, for which instant jamming did not take place, only the initial configurations were processed entirely.

Estimates for the number of beads detected by the watershed algorithm, the number of beads detected by merging 

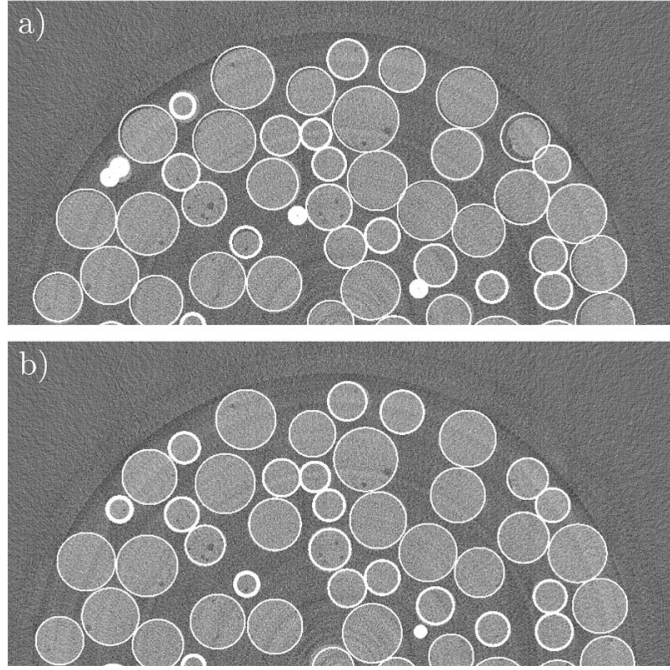

FIG. 7. Sphere fittings (white circles) superimposed on the original tomographs after (a) detection and (b) optimization.

sphere fittings corresponding to oversegmentations, as well as the number of beads detected by the user were calculated (Fig. 8). 98.5\% of the beads were detected by our procedure and the remaining $1.5 \%$ were estimated only by manual detection. After user correction $100 \%$ of the beads in the field of view were detected for all 3D images treated.

The total time needed to treat one 3D tomography image was approximately $20 \mathrm{~h}$, of which $25 \%$ corresponds to user work and the other $75 \%$ to computer calculations.

To estimate how precise the final sphere fittings (after optimization) are, a 3D binary rendition of the sphere fittings was created. This image was subtracted from the binary image obtained after preprocessing. The symmetric difference (Fig. 9) between these two images is an approximate measure of the total volume discrepancy between the beads and their sphere fittings.

Dividing this measure of discrepancy by the number of beads gives an approximate average for the volume error $\langle\Delta V\rangle$ affecting a bead sphere fitting. The corresponding re-

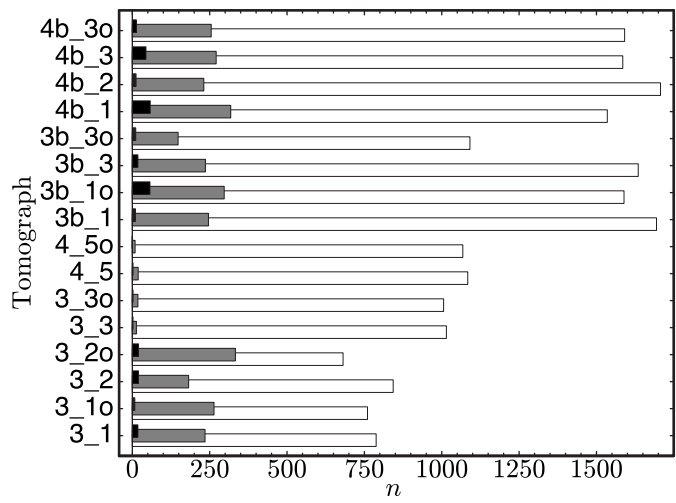

FIG. 8. Results of the detection: $n$, number of beads; tomographs are named after the corresponding simulation, the absence of the letter o indicates images taken before hole opening, a letter o indicates images taken after hole opening; white, correct detection; gray, automatically corrected for oversegmentation; black, corrected manually for undersegmentation and bad cuts.

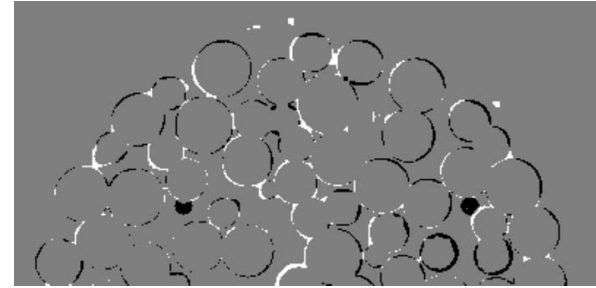

FIG. 9. Error estimation: symmetric difference of fitted spheres and preprocessed image (white shapes, particles in the preprocessed image absent in the sphere fittings; black shapes, opposite case).

sults for different tomographs are reported in Fig. 10. The average bead volume is around $0.07 \mathrm{~mm}^{3}$; thus the volume error affecting a bead is found to be between $15 \%$ and $20 \%$ of the average bead volume.

It is interesting, assuming that the real beads are spherical, to evaluate the error made while estimating their radii and positions. For an approximate assessment, we can assume that the real beads are spherical and that, as a starting point, there is no error for the positions. Then the volume discrepancy is

$$
\Delta V=\left|\frac{4}{3} \pi r_{\mathrm{SF}}^{3}-\frac{4}{3} \pi r_{B}^{3}\right|
$$

where $r_{\mathrm{SF}}$ and $r_{B}$ are, respectively, the radii of the sphere fitting and the real bead.

When $r_{\mathrm{SF}}$ and $r_{B}$ are close, the right-hand side of (1) is close to $4 \pi\left|r_{\mathrm{SF}}-r_{B}\right| r_{\mathrm{SF}} r_{B}$ so that

$$
\left|r_{\mathrm{SF}}-r_{B}\right| \simeq \frac{1}{4 \pi r_{\mathrm{SF}} r_{B}} \Delta V
$$

Taking $r_{\mathrm{SF}}$ and $r_{B}$ equal to the average bead radius $r_{a}(\simeq 250 \mu \mathrm{m})$, on the right-hand side of (2), the average error made on the bead radii can be estimated as $\langle\epsilon\rangle$ $=\frac{1}{4 \pi r_{\alpha}^{2}} \Delta V$. We found that the error on the distance between the centers of real beads and their sphere fittings can also be roughly estimated as $\langle\epsilon\rangle=\frac{1}{4 \pi r_{a}^{2}} \Delta V$. Values for this approximate average error measure are shown in Fig. 11. It shows

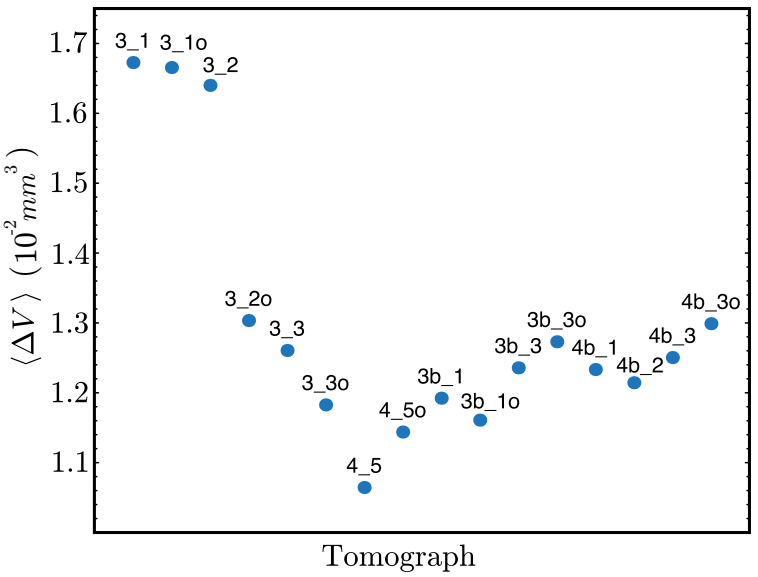

FIG. 10. (Color online) Average volume symmetric difference between a real bead and its sphere fitting. 


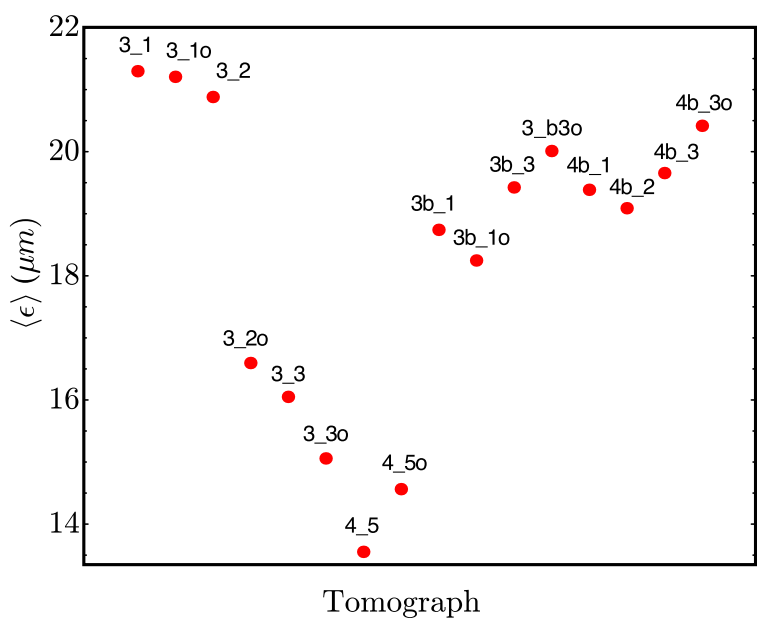

FIG. 11. (Color online) Average fitting error on positions and radii.

that the average error on the positions and the radii is found to be between $5 \%$ and $10 \%$ of the average radius.

\section{Reconstructing the exact shape and position of the container}

Due to manipulations between experiments the cylinder position varied. As a consequence, to simulate as realistically as possible the experiments, the radius of the cylinder and the position of the receptacle bottom had to be estimated. For this, the cylinder and its position were set to be the solution to an optimization problem.

To limit the calculations, we found the beads that were most probably touching the cylinder in the real experiments. This was done by starting with a rough estimation of cylinder radius and position and detecting spheres $\left\{S_{1}, \ldots, S_{n}\right\}$ in the sphere fitting close to this cylinder. For each sphere $S_{i}$, we call its center $c_{i}$ and its radius $r_{i}$. Let the radius of the cylinder be $R>0$ and its axis a line $a$; we solved the following optimization problem:

$$
\begin{gathered}
\text { minimize } \quad \sum \xi_{i}^{2} \\
\text { under constraints } \xi_{i}^{2}=\left[d\left(c_{i}, a\right)+r_{i}-R\right]^{2}, \\
R \in \mathcal{R}_{+}^{*}, \\
a \text { line in } \mathcal{R}^{3} .
\end{gathered}
$$

In other words, we found the cylinder for which a measure of the distance between a sphere fitting and the cylinder (given by $\left.\xi_{i}^{2}\right)$ is essentially small for all sphere fittings $\left(\sum \xi_{i}^{2}\right.$ is small).

A similar procedure was applied to estimate the receptacle bottom. MATHEMATICA optimization functions were used for these steps. The values found for the angles between the cylinder axis and the vertical direction of the tomographic images as well as the angles between the cylinder axis and the receptacle bottom normal varied between $0.1^{\circ}$ and $1.4^{\circ}$.
This is consistent with the fact that in the real experiment the cylinder and the receptacle bottom are separate pieces.

\section{NUMERICAL HOURGLASS EXPERIMENT SIMULATIONS WITH THE DEM}

As mentioned above, our strategy here is to use the initial data (fitted spheres along with the estimated cylindrical receptacle) corresponding to the observed physical hourglass experiments as the starting point of DEM simulations during which the bottom hole is opened as in the real experiment. This initial geometrical information does not fully characterize the medium, because it does not contain the information on the equilibrium forces. Therefore a preliminary DEM run starting from this situation is carried out, to allow the system to settle before opening the shutter. Appropriate DEM model parameters are found by trial and error, as will be explained. Once equilibrium is reached, a second DEM simulation with the opened shutter is run and the final situation is compared to the corresponding fitted final images from the physical experiment. How realistic is this coupled tomo-DEM technique? Can it reproduce the jamming and how closely can it predict the measured final configurations of the experiments? The objective of this study was to address these important questions. Rather than presenting the DEM approach in detail, we refer the reader to $[17]$ and $[18,32]$.

\section{A. Procedure}

In the first place, the DEM parameters had to be set to suit the needs of our simulation. The main parameters are the normal and tangential restitution coefficients, normal and tangential contact times, Coulomb friction coefficient, and simulation time step. The present study was performed with glass beads for which the Coulomb friction coefficient is about 0.95 .

Furthermore, a realistic value for the restitution coefficient is approximately 0.96 and for the contact time a realistic value would be about $1 \mu$ s. However, for simulation coherence, the simulation time step must be smaller than the contact time. Since small time steps like $1 \mu$ s lead to lengthy calculation times, the used contact time could not be selected to be realistic.

Another difficulty was the limited precision in bead radii and position estimates. Overlaps between beads or between beads and the receptacle caused by this lack of precision could possibly lead to unrealistic ejection at contact points. As a first step, we decided to pass over realism in the choice of individual DEM parameters in favor of a more realistic general behavior of the medium.

We used a dichotomic method in one experiment to find the restitution coefficient and contact time for which the movement of the beads in their initial rearrangement was realistically small. This procedure led to a restitution coefficient of 0.0011 and a contact time of $0.7 \mathrm{~ms}$. As pointed out above, these values do not correspond to realistic modeling of glass beads, and would better fit beads of a much softer material.

The experiment setup was not entirely within the field of view; beads below the level of the receptacle bottom and at 
TABLE II. Simulation characteristics: Name ( $S$ followed by the name of the corresponding experiment, followed by letter when necessary); number $n$ of beads; hole radius $\tau(=B / 2)$; normal restitution coefficient $e_{n}$; normal contact time $t_{n}$; Coulomb friction coefficient $\mu$.

\begin{tabular}{cccccc}
\hline \hline Name & $n$ & $\tau(\mathrm{mm})$ & $e_{n}$ & $t_{n}(\mathrm{~s})$ & $\mu$ \\
\hline$S 3 \_1$ & 1041 & 0.75 & 0.0011 & 0.0007 & 0.95 \\
$S 3 \_2$ & 1045 & 0.75 & 0.0011 & 0.0007 & 0.95 \\
$S 3 \_3$ & 1030 & 0.75 & 0.0011 & 0.0007 & 0.95 \\
$S 4 \_5$ & 1105 & 1.0 & 0.001 & 0.0004 & 0.95 \\
$S 3 b \_1$ & 1950 & 0.75 & 0.0011 & 0.0007 & 0.95 \\
$S 3 b \_3$ & 1889 & 0.75 & 0.0011 & 0.0007 & 0.95 \\
$S 4 b \_1$ & 1911 & 1.0 & 0.0011 & 0.0007 & 0.95 \\
$S 4 b \_2$ & 1950 & 1.0 & 0.0011 & 0.0007 & 0.95 \\
$S 4 b \_3 \_a$ & 1899 & 1.0 & 0.0011 & 0.0007 & 0.95 \\
$S 4 b \_3 \_b$ & 1899 & 1.0 & 0.0011 & 0.0007 & 1.35 \\
\hline \hline
\end{tabular}

the top of the setup were not estimated. As discussed in Sec. II, the hole opening in the real experiments was located not at the receptacle bottom level, but at the bottom of the smaller cylinder linked to the receptacle hole. In the simulations beads below the receptacle bottom level were immobilized during the first phase of the procedure (closed shutter) to model the setup as realistically as possible. Opening the hole was then simulated by freeing these beads.

\section{B. Results and discussion of the experiment simulations with the DEM}

Table II shows the parameters for ten of the performed simulations. Films of the simulations as well as of DEMexperiment comparisons may be accessed at [33]. In all of them, the time step was $10 \mu$ s. The hole was opened at 0.07 $s$ of simulation time.

Figure 12 shows snapshots of simulation $S 4 \_5$. Immobilized beads are shown in red. In the first $15 \mathrm{~ms}$, a rearrangement of the beads takes place. This is the initial stabilization.

Opening the shutter leads to a small flow of beads. However, the flow stops and at $0.3 \mathrm{~s}$ the medium is at rest. Most beads move only slightly from their initial positions as shown in Fig. 13.

Results of this kind were obtained for simulations $S 33_{-} 1$, $S 3 \_2, S 3 \_3, S 4 \_5, S 3 b \_1, S 3 b_{-} 3$, and $S 4 b \_3 \_b$. In those cases the DEM approach yields images close to the real experiments; see Figs. 14 and 15. However, this works only if jamming occurs upon slight motions of the beads.

For experiment $4 b_{-} 3$, only the simulation $S 4 b_{-} 3_{-} b$ that uses a higher friction coefficient reproduces jamming and not the standard simulation $S 4 b_{-} 3_{-} a$. This indicates that the accuracy of the simulation procedure can change from case to case. Additional evidence for this point is provided by simulation $S 3 b \_3$, where the simulated final configuration differs from the measured final configuration more than in the other six near-equilibrium simulations. See also Sec. V.

As to cases not near equilibrium, simulations $S 4 b_{-} 1$ and $S 4 b \_2$ yield results quite different from those above. The tomographs show that jamming did not occur with only small movement of the beads but with a substantial flow. In these two cases, we did not manage to reconcile the final simulated configurations with the experimental findings. More details on this problem will given in the following section.

\section{SENSITIVITY ANALYSIS}

To get a better idea about the scope of the presented approach an analysis of sensitivity to random data perturbations was carried out for all DEM simulations reported in Table II.

The initial estimated bead data of the various hourglass experiments as provided by tomography and image analysis, were successively subjected to three types-I, II, and III-of random perturbations of varying intensities, the first two concentrating on bead sizes and the third on their positions. As a measure of the perturbation intensity we used the square root $\epsilon$ of the mean square deviation of the radius or of the center position of the beads, whichever applied. In type I and II perturbations, we add independent and identically distributed (i.i.d.) Gaussian random values $\rho_{i} \sim N\left(\mu, \sigma^{2}\right)$ to the radii of the fitted beads (mean $\mu$ and variance $\sigma^{2}$ ). The mean square error of the perturbation becomes

$$
\epsilon^{2}=E\left[\rho_{i}\right]=\sigma^{2}+\mu^{2} .
$$

According to the parameter values chosen, such a perturbation may change the average sphere volumes. For a typical sphere of radius $r_{i}$ this change computes as follows:

$$
\begin{aligned}
\left\langle\Delta V_{i}\right\rangle & =\frac{4}{3} \pi\left[E\left(r_{i}+\rho_{i}\right)^{3}-r_{i}^{3}\right] \\
& =\frac{4}{3} \pi\left[3 r_{i}^{2} E\left(\rho_{i}\right)+3 r_{i} E\left(\rho_{i}^{2}\right)+E\left(\rho_{i}^{3}\right)\right] \\
& =\frac{4}{3} \pi\left[3 r_{i}^{2} \mu+3 r_{i}\left(\mu^{2}+\sigma^{2}\right)+\mu^{3}+3 \mu \sigma^{2}\right] .
\end{aligned}
$$

For a given set of beads with radii $r_{i}, \ldots, r_{n}$, the average change of volume is 


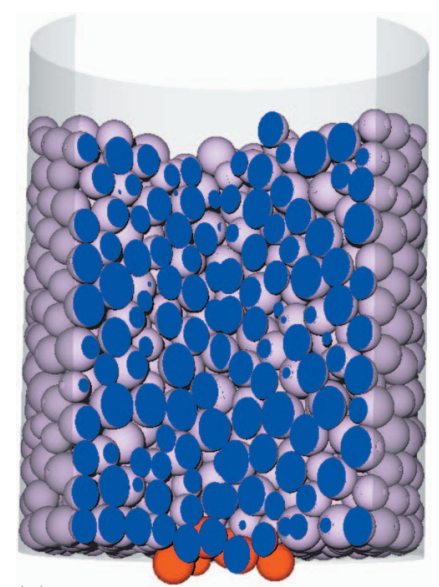

(a)

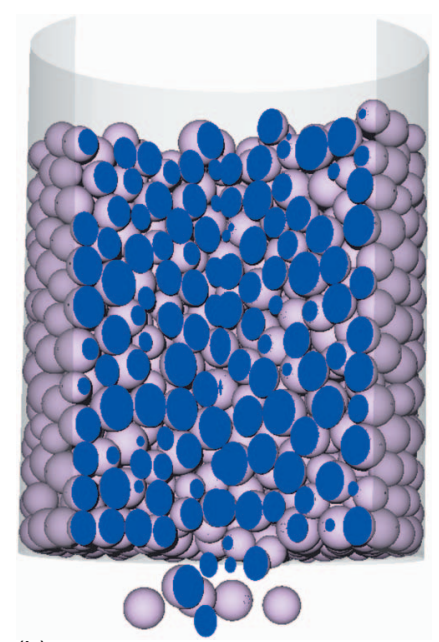

(b)

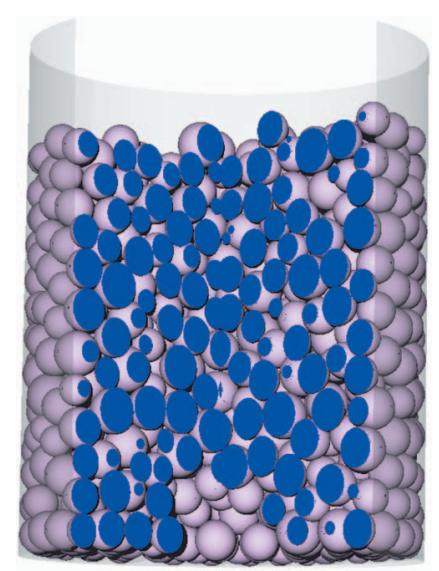

(c)

FIG. 12. (Color) Three snapshots of simulation $S 4 \_5$ at times (a) $0.0 \mathrm{~s}$ (shutter closed); (b) $0.08 \mathrm{~s}$ (intermediate stage); (c) $0.3 \mathrm{~s}$ (arched configuration).

$$
\langle\Delta V\rangle=\frac{4}{3} \pi\left[3\left\langle r^{2}\right\rangle \mu+3\langle r\rangle\left(\mu^{2}+\sigma^{2}\right)+\mu^{3}+3 \mu \sigma^{2}\right],
$$

where $\langle r\rangle$ and $\left\langle r^{2}\right\rangle$ are the mean and the mean squared radius of the original bead set.

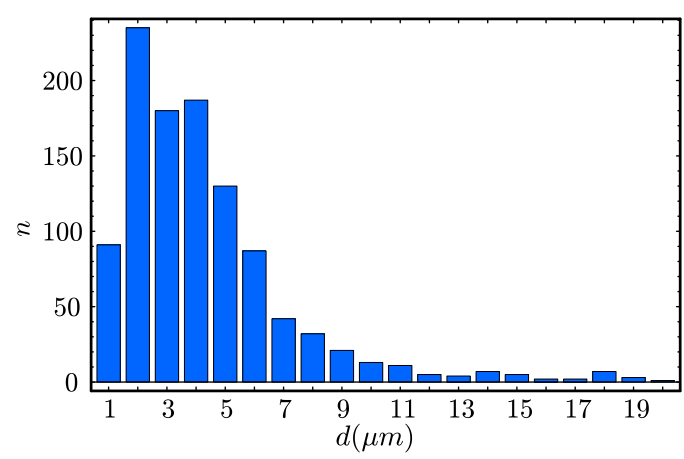

FIG. 13. (Color online) Histogram of bead displacements between 0.05 and $0.4 \mathrm{~s}$ for simulation $S 4$ _5 (outliers not shown).

In type I perturbations, we set $\mu=0$; thus there is no bias in the radius perturbations, which results in a mean volume increase $\langle\Delta V\rangle=4 \pi\langle r\rangle \sigma^{2}$. In type II perturbations we introduce a bias $\mu<0$ in order to correct for this volume increase. Thus, for a given intensity $\epsilon^{2}=E\left(\rho_{i}^{2}\right)$ we choose $\mu$ and $\sigma^{2}$ solving

$$
\begin{gathered}
\langle\Delta V\rangle=-2 \mu^{3}+3\left(\left\langle r^{2}\right\rangle+\epsilon^{2}\right) \mu+3\langle r\rangle \epsilon^{2}=0, \\
\epsilon^{2}=\mu^{2}+\sigma^{2} .
\end{gathered}
$$

Finally, type III (pure position) perturbations consist in adding i.i.d Gaussian random vectors $\gamma_{i} \sim N\left(0, \sigma^{2} I\right)$ (with uncorrelated coordinates of means 0 and variances $\sigma^{2}$ ) to the fitted spheres' center coordinate vectors $c_{i}$. Here we use $\epsilon$ $=\sqrt{E\left(\|\gamma\|^{2}\right)}=\sqrt{3} \sigma$ as a measure for the center perturbation intensity.

\section{A. Perturbation experiments}

For each simulation from Table II except simulations $S 4 b_{-} 1$ and $S 4 b_{-} 3_{-} a$, perturbations of types I, II, and III were run in succession, in each case varying the intensity $\epsilon$ between 10 and $100 \mu \mathrm{m}$, in steps of $5 \mu \mathrm{m}$. Two replications of each perturbation were run.

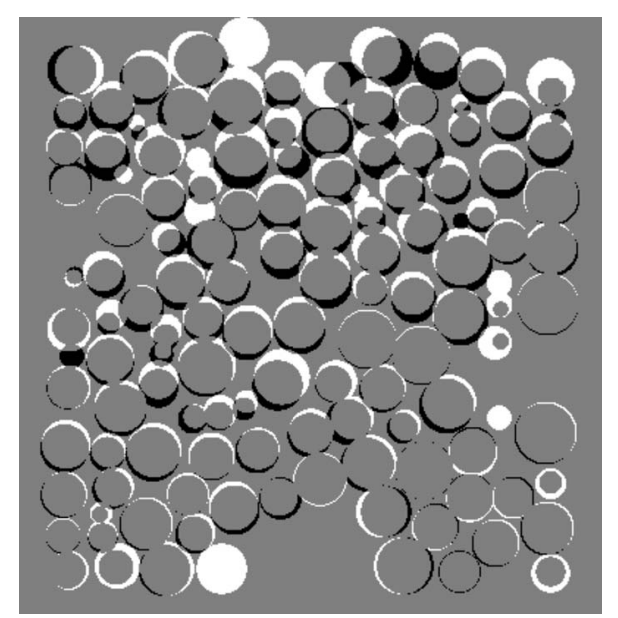

FIG. 14. Vertical cut highlighting the deviation between physical experiment 4_5 and simulation $S 4$ _5: white shapes, bead traces in the simulation not coinciding with their physical experiment counterparts; black shapes, opposite case. 


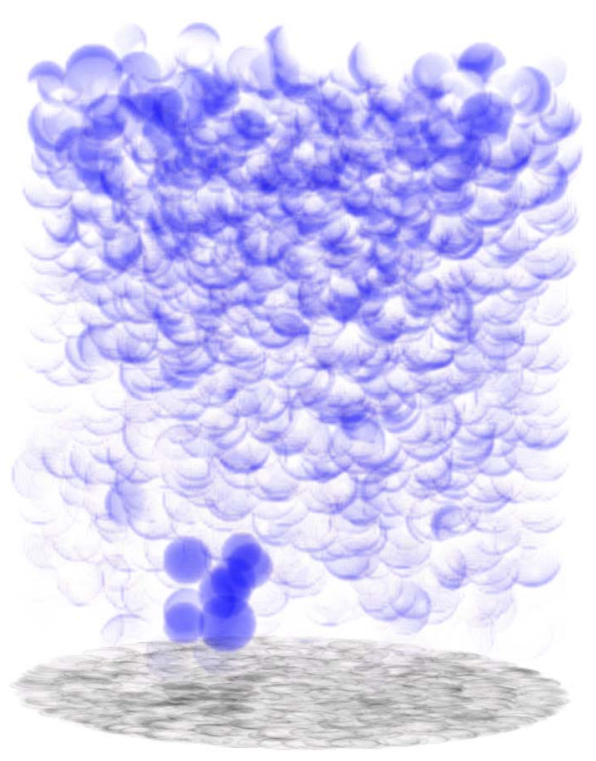

FIG. 15. (Color online) 3D rendered volume of the deviation between physical experiment 4_5 and simulation $S 4$ _5: bead traces in the physical experiment not coinciding with their simulation counterparts

In each case the simulations proceeded exactly as in the unperturbed case; see Sec. IV. That is, each set of perturbed data was stabilized by first running the DEM simulation for $0.07 \mathrm{~s}$ with the hole closed. The DEM simulation was then continued for 0.23 more seconds with the hole opened. The final configuration, after a total $0.3 \mathrm{~s}$ simulation time, was recorded. For each experiment, the parameters for the simulation model were the same as in Table II. The difference in behavior between perturbed and nonperturbed simulations at time $t$ is measured using $\eta_{t}$ given by the following norm:

$$
\eta_{t}=\sqrt{\sum_{i=1}^{n}\left\|\mathbf{x}_{t, i}^{P}-\mathbf{x}_{t, i}^{R}\right\|^{2}},
$$

where $\left\{\mathbf{x}_{t, i}^{P}\right\}_{i=1}^{n}$ are the center positions of the beads in the perturbed simulation at time $t$ and $\left\{\mathbf{x}_{t, i}^{R}\right\}_{i=1}^{n}$ are the center positions of the reference nonperturbed simulation at time $t$. Measurements of $\eta_{t}$ have been obtained after the initial settling $(t=0.07 \mathrm{~s})$ and for the final situation $(t=0.3 \mathrm{~s})$.

\section{B. Discussion of the sensitivity analysis}

Figure 16 represents results for some of the perturbation simulations carried out. We omit depicting the corresponding graphs for the remaining simulations since they are very similar to Fig. 16(a).

Sensitivity to size perturbations is very pronounced indeed. One can see that already with an intensity $\epsilon=10 \mu \mathrm{m}$, which is just slightly above the resolution of the image analysis (cf. Sec. III B), the perturbed settled system differs markedly from the unperturbed system. For size perturbation intensities $\epsilon \geq 30$, the deviation $\eta_{0.3 \mathrm{~s}}$ between perturbed and unperturbed settled systems grows by several orders of magnitude. On the other hand, the sensitivity to position perturbations is much smaller. Here data perturbations of intensity

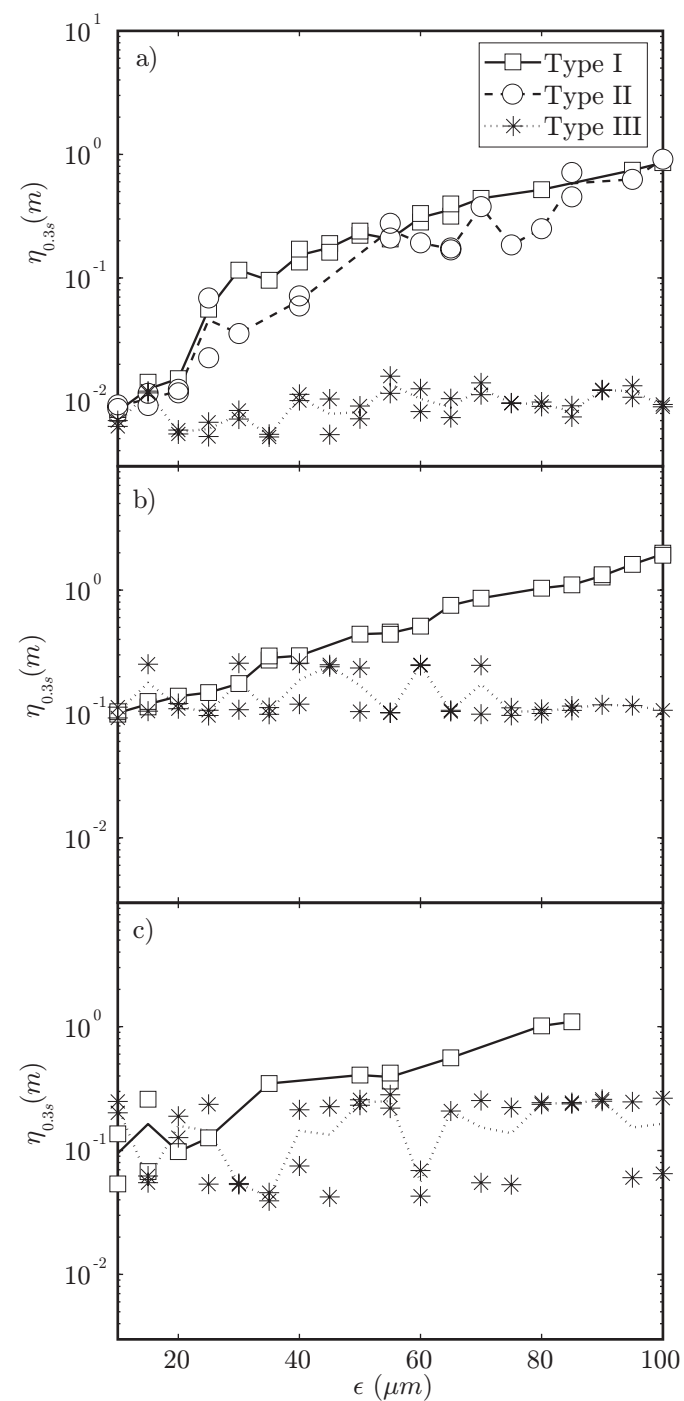

FIG. 16. Sensitivity analysis results showing the dominating effect of size vs position perturbations; $\eta_{0.3 \mathrm{~s}}(\epsilon)$ is given for experiments (a) 3_1, (b) $4 b_{-} 2$, and (c) $4 b_{-} 3$. Type II perturbations were carried out only for experiment 3_1. Lines represent the average value of the corresponding replications. Note that plots of types I and II coincide and dominate the plot of type III.

up to $\epsilon=100 \mu \mathrm{m}$ lead to essentially the same stabilized images as unperturbed data.

How can this very different response to size and location perturbations be explained? Perturbing data can be thought of as performing a certain amount of work, that is storing perturbation energy in the system. In the DEM, changing positions or sizes amounts to changing the spring forces acting between the spheres and between these and the receptacle walls. Figure 17 shows the amount of energy furnished by data perturbations of the three types to the system.

Observe that the energy from size perturbations is large for type I, where there is a mean volume increase. As expected, perturbations of the same intensity increasing the average volume require much more work than size perturbations keeping the volume constant. The latter turn out to require much the same work as position perturbations of an 


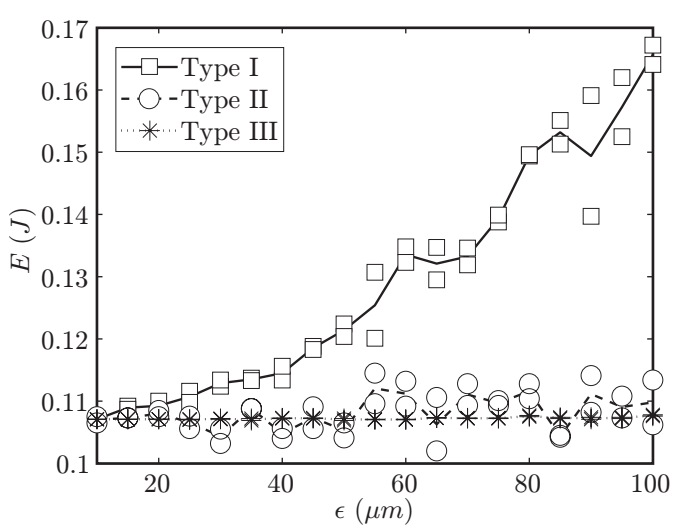

FIG. 17. Plots of $E(\epsilon)$, energy furnished to the system as a function of perturbation size, for experiment 3_1. Lines represent the average value of the corresponding replications. Note that the plot of type I dominates the coinciding plots of types II and III.

equivalent amount, which is what a simple geometric reasoning would lead one to surmise.

But this means that the large difference between the response to size perturbations and that to position perturbations cannot be explained by a difference in the amount of invested perturbation energy. Now observe that, when perturbing data, the new system will develop forces driving it in a direction opposite to the perturbations. In the case of position perturbations the system will develop restitutive forces driving it back to the unperturbed situation, provided the perturbation is not too large. On the other hand, whenever sizes are perturbed, there is normally no equilibrium configuration for the new spheres in the vicinity of their old positions. Hence the stored deformation energy cannot drive the system back to the old set of positions, but will drive the system to a new equilibrium.

Figure 16(b) shows the sensitivity to perturbations for experiment $4 b \_2$ for which jamming took place after a substantial flow. Such a flow occurred also for the corresponding nonperturbed and perturbed simulations and sensitivity to perturbations is approximately ten times higher than for instant-jamming experiments even for small perturbations $(\sim 10 \mu \mathrm{m})$. This shows that correctly simulating the experiment, with its substantial flow, is not possible with the means at hand. In this case, simulations show a chaotic behavior, i.e., a high sensitivity to small data perturbations.

A large flow of beads also occurred for perturbed simulations of experiment $4 b_{-} 3$ [Fig. 16(c)]. These two cases showed a very high sensitivity, not only to size but also to location perturbations. This fact indicates that the associated initial configurations are unstable. For such cases, where the motion quickly becomes chaotic, tomo-DEM presently attains its limit.

\section{CONCLUSION}

We have established the viability of tomo-DEM, an approach to exploring the microstructure of a polydisperse granular medium coupling $\mathrm{x}$-ray tomography and DEM simulations through image analysis, pointing to both its potential and its limitations. When applied to jamming in an hourglass, the technique gives accurate predictions of real experiments in most cases of instant jamming, while-at least in its present form-it tends to attain its limits when the systems are unstable. Very careful image analysis producing accurate geometry information turned out to be crucial. A sensitivity analysis to random data perturbations further brought to light an interesting phenomenon. The position perturbation energy fed into the system tends to drive it back to the unperturbed equilibrium while the size perturbation energy drives it away from it.

The fact that the first instants of the granular behavior can be faithfully reproduced by the tomo-DEM technique demonstrates the short-range predictive power of the approach when applied near equilibrium. This leads to a broad range of applications of the tomo-DEM approach in the study of granular media, using the DEM as an interpolating device between successive $\mathrm{x}$-ray snapshots.

\section{ACKNOWLEDGMENT}

We gratefully acknowledge access to the NSRRC synchrotron facility in Taiwan.
[1] L. Pournin, M. Ramaioli, P. Folly, and Th. M. Liebling, Eur. Phys. J. E 23, 229 (2007).

[2] J. D. Bernal, Nature (London) 183, 141 (1959); Proc. R. Soc. London, Ser. A 280, 299 (1964).

[3] J. D. Bernal and G. Mason, Nature (London) 188, 910 (1960); J. D. Bernal, G. Mason, and K. R. Knight, ibid. 194, 957 (1962); J. D. Bernal, I. A. Cherry, J. L. Finney, and K. R. Knight, J. Phys. E 3, 388 (1970).

[4] G. D. Scott, Nature (London) 188, 908 (1960); R. Rutgers, ibid. 193, 465 (1962); G. D. Scott and D. M. Kilgour, Jr., Br. J. Appl. Phys. 2, 863 (1969).

[5] J. L. Finney, Proc. R. Soc. London, Ser. A 319, 479 (1970).

[6] P. Spanne, J. F. Thovert, C. J. Jacquin, W. B. Lindquist, K. W. Jones, and P. M. Adler, Phys. Rev. Lett. 73, 2001 (1994).
[7] C. A. Baldwin, A. J. Sederman, M. D. Mantle, P. Alexander, and L. F. Gladden, J. Colloid Interface Sci. 181, 79 (1996).

[8] W. B. Lindquist, S.-M. Lee, D. A. Coker, K. W. Jones, and P. Spanne, J. Geophys. Res. 101, 8297 (1996).

[9] A. J. Sederman, P. Alexander, and L. F. Gladden, Powder Technol. 117, 255 (2001).

[10] R. I. Al-Raoush and C. S. Willson, J. Hydrol. 300, 44 (2005).

[11] G. T. Seidler, G. Martinez, L. H. Seeley, K. H. Kim, E. A. Behne, S. Zaranek, B. D. Chapman, S. M. Heald, and D. L. Brewe, Phys. Rev. E 62, 8175 (2000).

[12] P. Richard, P. Philippe, F. Barbe, S. Bourlès, X. Thibault, and D. Bideau, Phys. Rev. E, 68, 020301(R) (2003).

[13] T. Aste, M. Saadatfar, A. Sakellariou, and T. J. Senden, Physica A 339, 16 (2004). 
[14] M. Saadatfar, A. Kabla, T. J. Senden and T. Aste, in Proceedings of the Conference on Powders and Grains, Stuttgart, Germany, 2005, edited by R. García-Rojo, H. J. Herrmann, and S. McNamara (Taylor and Francis Group, London, 2005), p. 33.

[15] X. Fu, M. Dutt, A. C. Bentham, B. C. Hancock, R. E. Cameron, and J. A. Elliott, Powder Technol. 167, 134 (2006).

[16] J. Lambert, I. Cantat, R. Delannay, A. Renault, F. Graner, J. A. Glazier, I. Veretennikov, and P. Cloetens, Colloids Surf., A 263, 295 (2005).

[17] P. A. Cundall and O. D. L. Strack, Geotechnique 29, 47 (1979).

[18] J.-A. Ferrez and Th. M. Liebling, Philos. Mag. B 82, 905 (2002).

[19] J. J. Moreau, Eur. J. Mech. A/Solids 13, 93 (1994).

[20] D. Müller, Ph.D. thesis no. 1545, EPFL, 1996.

[21] S. Luding, Ph.D. thesis, Universität Freiburg, 1994.

[22] C. R. A. Abreu, F. W. Tavares, and M. Castier, Powder Technol. 134, 167 (2003).

[23] L. Pournin, M. Weber, M. Tsukahara, J.-A. Ferrez, M. Ra- maioli, and Th. M. Liebling, Granular Matter 7, 119 (2005).

[24] M. Ramaioli, L. Pournin, and Th. M. Liebling, Phys. Rev. E 76, 021304 (2007).

[25] W. L. Tsai, P. C. Hsu, Y. Hwu, J. H. Je, Y. Ping, H. O. Moser, A. Groso, and G. Margaritondo, Nucl. Instrum. Methods Phys. Res. B 199, 436 (2003).

[26] S. Baik, H. S. Kim, M. H. Jeong, C. L. Lee, J. H. Je, Y. Hwu, and G. Margaritondo, Rev. Sci. Instrum. 75, 4355 (2004).

[27] Y. Hwu et al., Biophys. J. 87, 4180 (2004).

[28] J. B. T. M. Roerdink and A. Meijster, Fund. Inform. 41, 187 (2001).

[29] L. Vincent and P. Soille, IEEE Trans. Pattern Anal. Mach. Intell. 13(6), 583 (1991).

[30] S. Al-Thyabat and N. J. Miles, Powder Technol. 166, 3 (2006).

[31] W. S. Rasband, computer code IMAGEJ (U.S. National Institutes of Health, Bethesda, MD, 2006), http://rsb.info.nih.gov/ij/

[32] J.-A. Ferrez, Ph.D. thesis no. 2432, EPFL, 2001.

[33] http://ima.epfl.ch/ tsukahar/public-data 